\title{
Growth of high purity semiconductor epitaxial layers by liquid phase epitaxy and their characterization
}

\author{
S DHAR \\ Department of Electronic Science, University of Calcutta, Kolkata 700 009, India
}

\begin{abstract}
This paper briefly describes our work and the results on the growth of several III-V epitaxial semiconductor materials in high purity form by liquid phase epitaxy (LPE) technique. Various possible sources of impurities in such growth are listed and step-by-step procedures adopted to reduce them are discussed in particular reference to the growth of GaAs layers. The technique of growing very high purity layers by treating the melt with erbium is described for the growth of InGaAs and GaSb layers.
\end{abstract}

Keywords. Growth; epitaxy; characterization; III-V compounds.

\section{Introduction}

Liquid phase epitaxy (LPE) technique is widely used for the growth of composite semiconductor layer structures for the manufacture of optoelectronic devices, viz. light emitting diodes, lasers and photodiodes. The successful operation of these devices strongly depend on the quality of the epitaxial layers. The growth system must be able to grow materials with very low unintentional impurity density, high mobility and good luminescence properties. We have used LPE technique to perfect the growth of a number of group III-V epitaxial materials such as GaAs, $\mathrm{GaAsSb}$, InGaAs, InGaP and $\mathrm{GaSb}$ in highly purified form. In this paper, we describe our experiences with the purification of GaAs and InGaAs through a number of modifications in the growth process and subsequent characterization.

\section{Sources of impurities during LPE growth}

LPE growth is usually carried out from supersaturated melts composed of some source materials, which are contained in a graphite boat. The boat is again placed inside a quartz reactor tube and growth is carried out at high temperatures under hydrogen gas flow. During this process, several kinds of metallic and non-metallic impurities may be introduced in the grown layer from the following possible sources: (a) The source materials themselves and the chemicals and reagents used to clean them; (b) the walls of the growth boat which comes in contact with the melt; (c) contaminants deposited on the inner walls of the quartz reactor tube which are transported to the melt by the ambient gas, during high temperature growth; (d) the carrier hydrogen gas itself and the impurities carried by

sdhar_25@yahoo.co.in the gas from the inner walls of the gas lines and (e) the containers for storing, handling and cleaning of the materials and the substrate.

In order to minimize the impurities coming from the above sources, the following precautions are taken: (i) the materials for growth are taken in high purity form of $99.9999 \%$ or even better. Source of purchase is important. The reason is that the manufacturer specifies the total residual impurity in a particular material which is the sum of the various individual metallic and non-metallic impurities, present in the same. Some of the impurities are electrically active which can dope the material, $n$ - or $p$-type, and some are non-active which do not alter the electrical or optical properties of the same. Certain manufacturers provide materials where the percentage of electrically active impurities is less in proportion and they are suited for epitaxial growth. The materials, before loading into the growth furnace, are thoroughly degreased in organic solvents, followed by etching in an inorganic acid or alkali. This step ensures that the contaminated surface regions of the growth material are removed. Very high purity reagents are to be used so that they themselves do not introduce new impurities. The cleaned and etched materials are transferred to the LPE furnace and baked for $20-30 \mathrm{~h}$ at high temperatures under ultrapure hydrogen flow. During baking, traces of dissolved oxygen in the growth materials are slowly reduced by hydrogen to water vapour and some of the volatile impurities are also evaporated out in the process. Baking of LPE melt is an established procedure to get very low carrier concentration semiconductor layers; (ii) the LPE growth boat is made of ultra high purity low porosity graphite. Low porosity helps to keep the absorbed gases to a minimum. From time to time, the boat is fired in vacuum at 1400 $1600^{\circ} \mathrm{C}$ to remove the absorbed impurities; (iii) the reactor tube is always made of high purity quartz and, before every use, the inside of the tube is cleaned and etched in 
HF based reagents, followed by high temperature baking. This process helps to remove the impurities, which are deposited on the inner surfaces of the reactor tube during growth; (iv) Pd-diffused hydrogen gas is used as the growth ambient. The gas lines are normally made of 316 stainless steel. Before installation, the inside of the tubes and valves are cleaned and passivated and (v) Pyrex or corning glass containers are used for cleaning the source materials and the substrate. These containers are, from time to time, treated with hot aqua regia and washed in $18 \mathrm{M} \Omega$ deionized water.

In addition to taking the above precautions, the loading of LPE boat is usually done in a class 1000 clean bench or preferably in a nitrogen glove box.

\section{Growth of GaAs layers}

We have grown GaAs layers using 99.9999\% polycrystalline GaAs dissolved in Ga. Both the materials were obtained from Johnson-Matthey Chemicals, UK. Degreasing of $\mathrm{Ga}$ and $\mathrm{GaAs}$ was done by successive rinsing in EL grade trichloroethylene, acetone and methanol, followed by washing in $18 \mathrm{M} \Omega$ deionized water. Finally, Ga was etched in $\mathrm{HCl}$ and $\mathrm{GaAs}$ in $5 \mathrm{H}_{2} \mathrm{SO}_{4}+1 \mathrm{H}_{2} \mathrm{O}_{2}+1 \mathrm{H}_{2} \mathrm{O}$ solution. Ga was loaded in two bins of the growth boat, one for the growth melt and other for the etch melt and baked at $780^{\circ} \mathrm{C}$ for $20 \mathrm{~h}$ under $\mathrm{Pd}$-diffused hydrogen obtained from a hydrogen gas generator. Growth was done on semi-insulating or $n^{+}$GaAs substrates, oriented along the $<100>$ direction, which were degreased and etched in $5 \mathrm{H}_{2} \mathrm{SO}_{4}+1 \mathrm{H}_{2} \mathrm{O}_{2}+1 \mathrm{H}_{2} \mathrm{O}$ solution, followed by mild etch in $1 \% \mathrm{Br}_{2}$ in methanol. Layers up to $8 \mu \mathrm{m}$ thick were grown at $750-760^{\circ} \mathrm{C}$ under a supersaturation of $5-6^{\circ} \mathrm{C} / \mathrm{min}$. After each growth run, the mobility and carrier concentration of the layers were measured by Van der Pauw Hall measurements. At the beginning of GaAs growth, typical impurity (donors and acceptors) concentrations of above $10^{17} \mathrm{~cm}^{-3}$ and mobility of 3000 and $9600 \mathrm{~cm}^{2} / \mathrm{V} \cdot \mathrm{s}$ at 300 and $77 \mathrm{~K}$, respectively, were obtained. The very

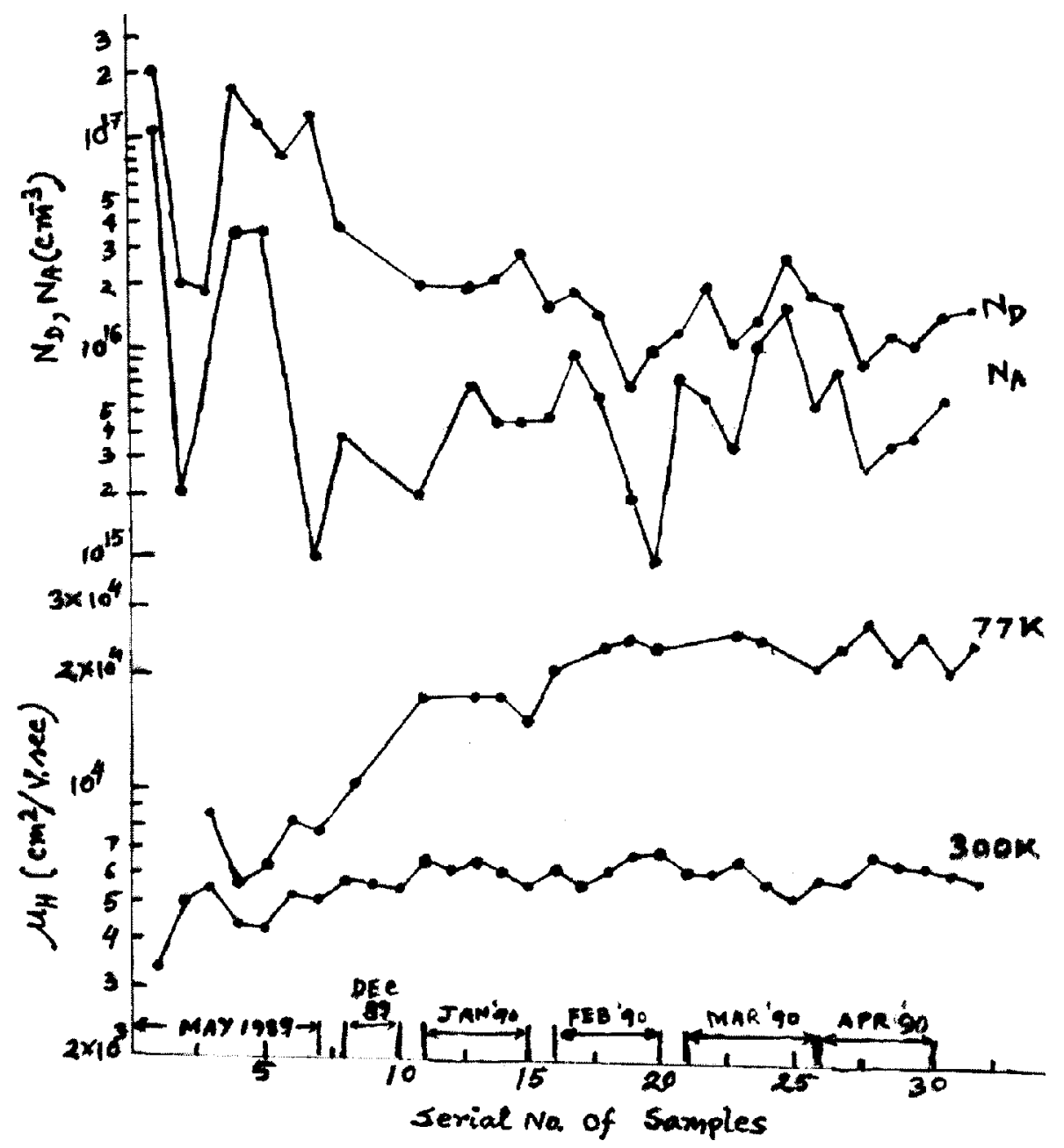

Figure 1. Gradual improvement of LPE layer quality in the author's laboratory over the initial period of one year. 
low value of $77 \mathrm{~K}$ mobility indicated that the layers were heavily compensated. Through continuous and systematic improvement of the process with the aim of reducing the residual impurities, the layer quality improved significantly. Figure 1 shows the gradual improvement of the impurity concentration and mobility of our LPE-grown GaAs layers over a period of one year. We have further used controlled addition of In metal to GaAs melt to marginally reduce the impurity density and to grossly decrease the crystalline defects. In, added to GaAs melt is incorporated in the grown layer as isoelectronic dopant. Figure 2 shows the result of In addition to GaAs. It is apparent that the dislocation density, measured by etch pit density (EPD) technique is greatly reduced by an optimum In doping. Details of this work has been described elsewhere (Dhar et al 1991). We have also shown that such doping is quite effective in reducing the concentration of the native deep level traps (Mallik and Dhar 1994; Mallik et al 1994), although the isoelectronic dopant itself may generate new traps (Dhar et al 1995).

\section{Growth of InGaAs layers}

Lattice matched InGaAs layers, grown over InP substrates, is one of the most important materials in group III-V electronic and optoelectronic devices. We have grown this material using the composition of polycrystalline InAs and GaAs, dissolved in In solvent. These materials in $99.9999 \%$ purity were imported from Johnson-

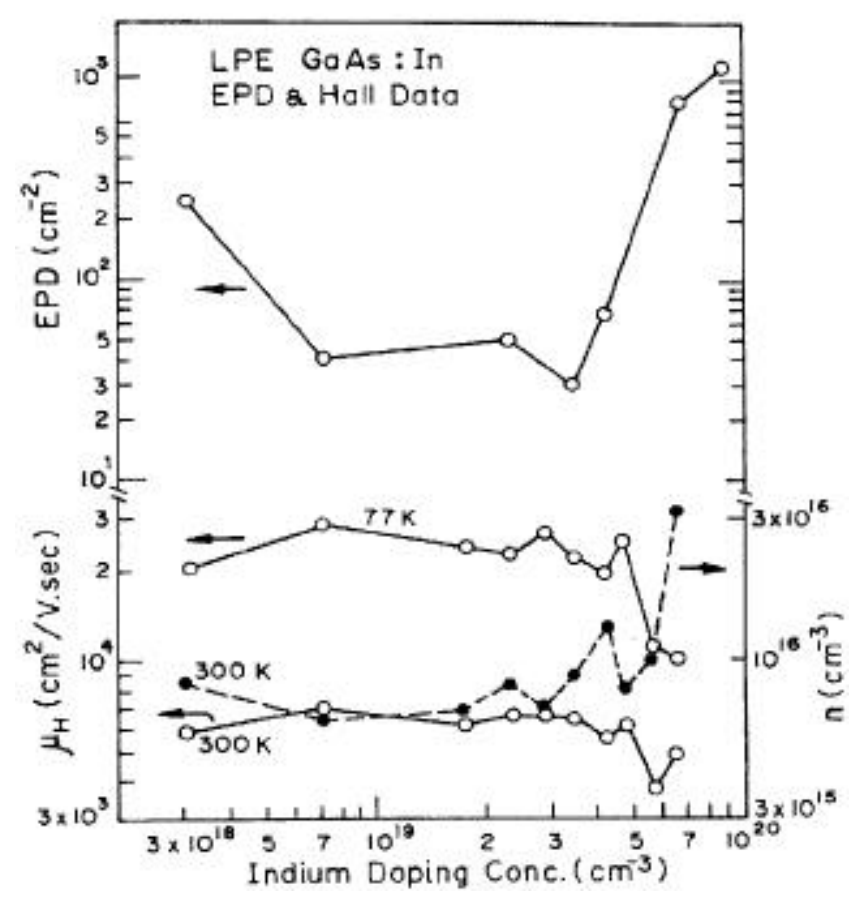

Figure 2. Change of dislocation density and transport parameters of LPE GaAs layers with In doping.
Matthey Chemicals, UK. Cleaning and etching of the materials, prior to growth, were done in a process similar to that for GaAs. In metal alone was baked in LPE furnace at $750-760^{\circ} \mathrm{C}$ for $20 \mathrm{~h}$ followed by melt bake at $700-720^{\circ} \mathrm{C}$ for the same period. Growth was typically done at $645-647^{\circ} \mathrm{C}$ under a melt supersaturation of 2$3^{\circ} \mathrm{C}$. During growth, the melt was constantly cooled at $0 \cdot 1{ }^{\circ} \mathrm{C} / \mathrm{min}$ to keep the melt supersaturated. The carrier concentrations of the grown layers were in the range (2$3) \times 10^{15} \mathrm{~cm}^{-3}$ and the Hall mobilities were 11000 and $35000 \mathrm{~cm}^{2} / \mathrm{V} \cdot \mathrm{s}$ at $300 \mathrm{~K}$ and $77 \mathrm{~K}$, respectively.

\section{Purification by Er doping}

Er and other metals, belonging to the rare earth family of the periodic table, have great affinity towards oxygen and other group VI elements which are usually present in the growth melt as impurities. When minute amounts of a rare earth metal is added to the LPE melt and heated to high temperatures, the rare earth forms insoluble complexes with these elements and separates out from the melt. This results in a major purification of the melt and very low carrier concentration layers are grown (Bantien et al 1987; Wu et al 1991; Gao et al 1996). We have grown InGaAs layers from melts containing up to $0.18 \mathrm{wt} \% \mathrm{Er}$ (Dhar et al 1997). In all cases, melt was prepared after the usual bake and then Er was added and baked again at $750-760^{\circ} \mathrm{C}$ for $5-6 \mathrm{~h}$. In figure 3 , we show the dramatic decrease of carrier concentration and increase in layer mobility after Er treatment of the melt. Purification of layer is also reflected in the very low full-width-at-halfmaximum (FWHM) of the photoluminescence (PL) spec-

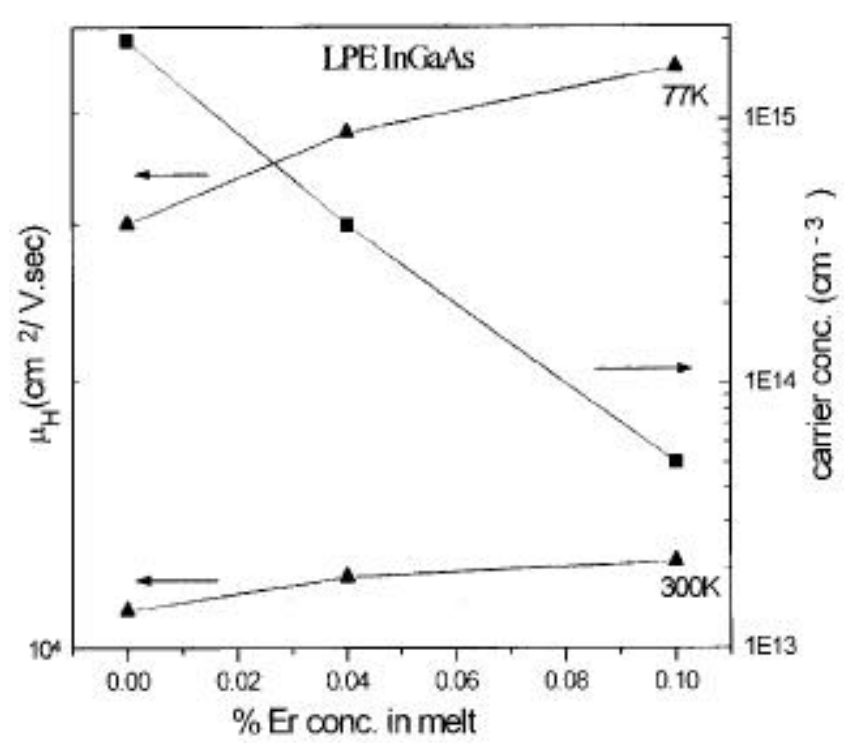

Figure 3. Decrease of carrier concentration and increase of mobility of LPE InGaAs with increase of Er content in the growth melt. 


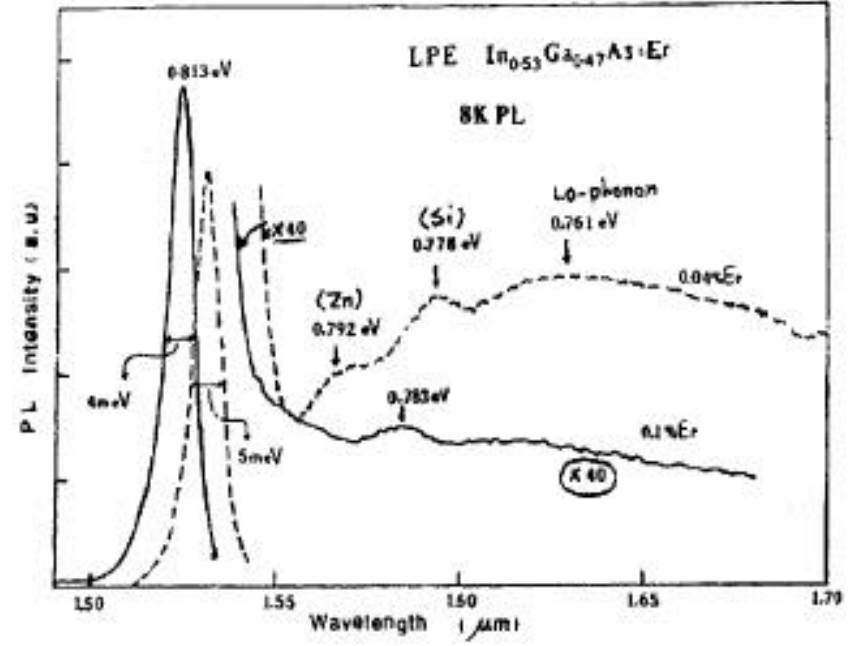

Figure 4. Photoluminescence spectrum of InGaAs layers, grown from Er-treated melts.

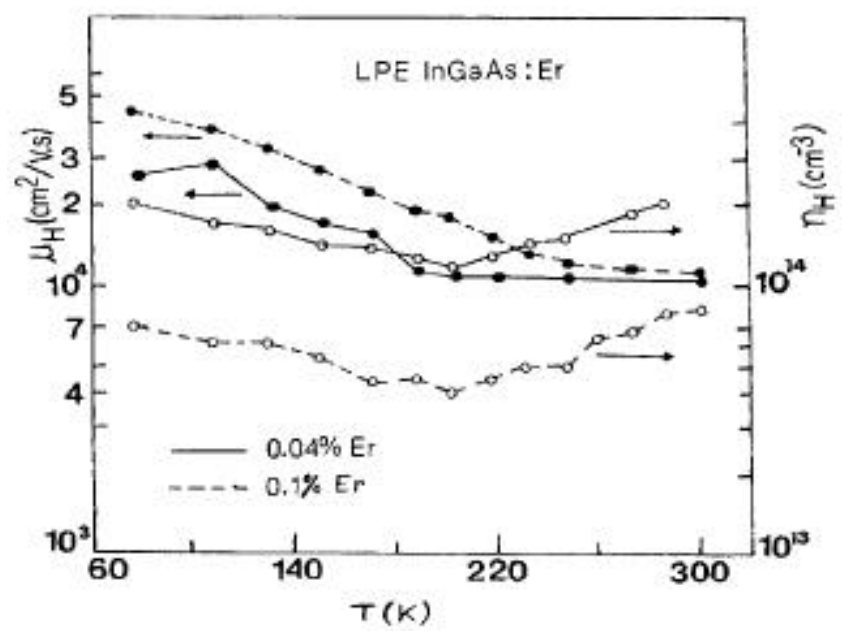

Figure 5. Temperature-dependent variation of carrier concentration and mobility in LPE InGaAs, grown from melts containing Er.

trum of the sample, shown in figure 4 . We have further measured the mobility of the layer in the temperature range $77-300 \mathrm{~K}$ (figure 5) and analysed the results by a theoretical curve fitting procedure. From the analysis, we got the values of the donor and acceptor concentrations for each sample. We have noted that as Er doping in the melt is increased, the compensation of the grown layer is also increased (Dhar et al 2000). This indicated that Er is more effective in removing the donor type impurities present in the melt.

Purification of LPE layers, through Er treatment of the growth melt, has been used for the growth of high purity $\mathrm{GaSb}$ layers. GaSb layers were grown from melts containing $\mathrm{GaSb}$ in $\mathrm{Ga}$. Initially $\mathrm{Ga}$ metal was baked at $750^{\circ} \mathrm{C}$ for $10 \mathrm{~h}$. Then the temperature of $\mathrm{Ga}$ was reduced

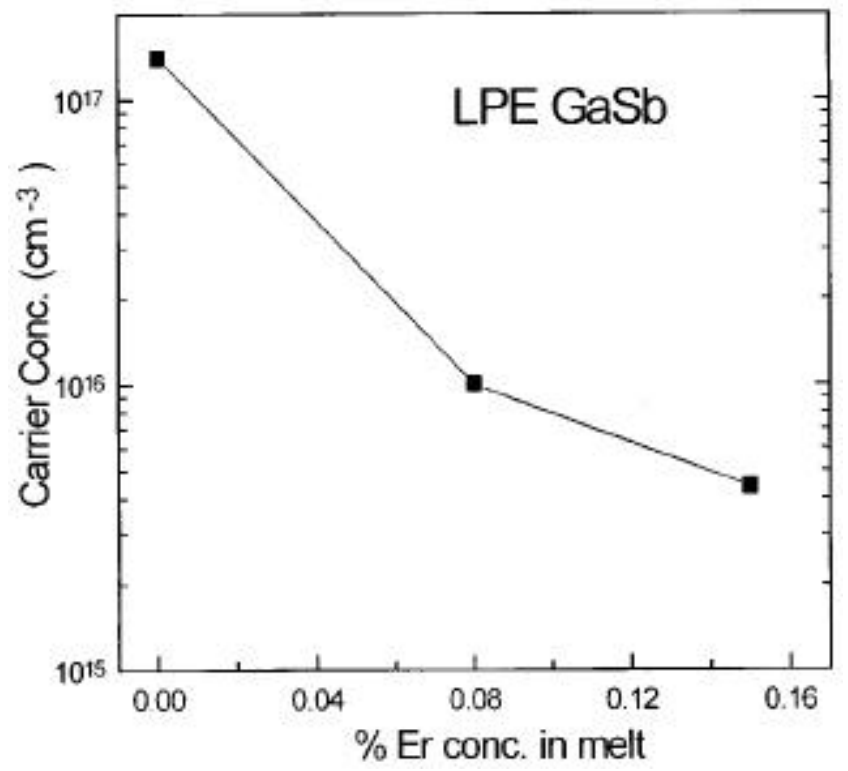

Figure 6. Reduction of carrier concentration of GaSb layers, grown from melts containing Er.

to around $480^{\circ} \mathrm{C}$ and equilibrated there. Ga at $480^{\circ} \mathrm{C}$ was then placed over an undoped single crystal wafer of $\mathrm{GaSb}$ and held there for $2 \mathrm{~h}$ till a saturated melt was prepared. The saturated melt was then baked at $650^{\circ} \mathrm{C}$ for $10 \mathrm{~h}$, followed by adding required amount of Er to the melt and baking again for $3 \mathrm{~h}$. GaSb layer growth was done on $n^{+}$ $<100>\mathrm{GaSb}$ substrates at around $470^{\circ} \mathrm{C}$ under a melt supersaturation of $6-7^{\circ} \mathrm{C}$ and a cooling ramp of $0.3-$ $0 \cdot 4^{\circ} \mathrm{C} / \mathrm{min}$. Carrier concentration in the grown layer was measured by capacitance-voltage technique on gold Schottky barrier diodes fabricated on the layer surface. Figure 6 shows the reduction of impurity concentration in the layers by about two orders of magnitude after addition of Er to the growth melt. Hence it is apparent that Er treatment provides an effective route for impurity reduction in LPE grown GaSb.

\section{Acknowledgements}

The work was funded by the Department of Information Technology, Ministry of Communications and Information Technology (formerly Department of Electronics), Government of India, through several research projects.

\section{References}

Bantien F, Banser E and Weber J 1987 J. Appl. Phys. 61 2803

Dhar S, Mallik K and Nag B R 1991 J. Appl. Phys. 693578

Dhar S, Mallik K and Mazumdar M 1995 J. Appl. Phys. 771531 
Dhar S, Paul S, Mazumdar M and Banerjee S 1997 J. Appl. Phys. 812391

Dhar S, Paul S and Kulkarni V N 2000 Appl. Phys. Lett. 761588

Gao W, Berger P R, Ervin M H, Pamulapati J, Lareau R T and Schauer S 1996 J. Appl. Phys. 807094
Mallik K and Dhar S 1994 Phys. Status Solidi (b) 184393

Mallik K, Dhar S and Sinha S 1994 Semicond. Sci. Technol. 9 1649

Wu M C, Chen E H, Chin T S and Kuang Y 1991 Jpn. J. Appl. Phys. 302679 